\title{
Postoperative laryngoscopy in thyroid surgery: proper timing to detect recurrent laryngeal nerve injury
}

\author{
Gianlorenzo Dionigi • Luigi Boni • Francesca Rovera • \\ Stefano Rausei • Paolo Castelnuovo • Renzo Dionigi
}

Received: 30 September 2009 / Accepted: 23 November 2009/Published online: 15 December 2009

(C) Springer-Verlag 2009

\begin{abstract}
Background There is currently a lack of consensus to support the proper timing for postoperative laryngoscopy that is reliable to diagnose recurrent laryngeal nerve palsy (RLNP) after thyroid surgery. The purpose of this study was to investigate the impact of different time intervals of fiber-optic nasolaryngoscopy (FNL) on the diagnosis of RLNP.

Method FNL was performed postoperatively at day $0(T 1)$, at second day post-op (T2), and +2 weeks (T3). For patients with RLNP, repeated examinations were performed at +2 (T4), +6 (T5), and +12 months (T6).

Results Four hundred thirty-four patients appear for postoperative FNL, providing 825 nerves at risk. Permanent RLNP occurred in $0.7 \%$, temporary RLNP in $6.7 \%$. RLNP rate was $6.4 \%$ at $T 1,6.7 \%$ at $T 2,4.8 \%$ at $T 3,2.5 \%$ at $T 4$, $0.8 \%$ at $T 5$, and $0.7 \%$ at $T 6$. Full recovery of vocal cord function was confirmed after rehabilitation in $87.5 \%$ of cases at $T 5$ and $89 \%$ in $T 6 . T 2$ was significantly superior to $T 3$ in terms of diagnosis of RLNP $(P<0.05)$. Of patients at $T 2,10.7 \%$ did not see any reason to FNL because of their normal voice register.

Conclusion FNL is essential for the detection of vocal cord paralysis after thyroidectomy. We report different time evaluation criteria of vocal cord motility with great and significant variability of results. Second day post-op inspection of the larynx (T2) is suggested. Symptomatic voice assessment is insufficient.
\end{abstract}

G. Dionigi $(\bowtie) \cdot$ L. Boni $\cdot$ F. Rovera $\cdot$ S. Rausei $\cdot$

P. Castelnuovo $\cdot$ R. Dionigi

Endocrine Surgery Research Center, Department of Surgical

Sciences, University of Insubria (Varese-Como),

Via Guicciardini 21100,

Varese, Italy

e-mail: gianlorenzo.dionigi@uninsubria.it
Keywords Thyroidectomy · Morbidity · Recurrent laryngeal nerve $\cdot$ Laryngoscopy

\section{Introduction}

Injury to the recurrent laryngeal nerve (RLN) post-thyroid surgery is the main cause of vocal cord paralysis associated with hoarseness, impaired voice register, dysphonia, dysphagia, and aspiration [1].

Reports document rates in the $1 \%$ to $20 \%$ range [2-4]. RLN paralysis rate is higher for cancer, Graves' disease, reoperation, substernal goiter, in less experienced surgical centers, and in patients in whom the inferior laryngeal nerve could not be identified and/or monitored during operation [2-5].

Many authors agree that the true overall injury rate of RLN is not yet delineate for several actual reasons: (a) no routine audit by centers performing thyroid surgerynot all patients in fact undergo a systematic laryngeal examination in the postoperative period $[6,7]$; (b) units with unfavorable data are less likely to report their data [7]; (c) absence of data in literature on bilateral RLN paralysis incidence [7]; (d) lack of reliability of clinical symptoms in vocal cord paralysis. Symptomatic assessment of vocal cord paralysis is notoriously inaccurate, and unilateral laryngeal paralysis can sometimes be completely asymptomatic. So, too many patients with postoperative hoarness may not have vocal cord paralysis [8,9]; and (e) there is considerable variation in the reported frequency of RLN palsy rates after thyroid surgery due to the different methods of diagnosing RLN palsy (indirect laryngoscopy versus fiber-optic nasolaryngoscopy (FNL) versus videostroboscopy), each of which have significant different sensitivities and specificities [10]. 
Assessment of the vocal cord function (VCF) with a laryngoscope is of great importance in the preoperative and postoperative evaluation of patients undergoing thyroidectomy [7]. Preoperatively, VCF detects an existing RLN palsy from local invasion of the nerve by extrathyroidal cancer extension or regional lymph node metastases $[8,11$, 12]. Postoperative assessment of VCF document a wellpreserved VCF, or an iatrogenic RLN injury, and yet the benefits of speech, rehabilitation, steroid, and surgical treatment [7].

There is currently a lack of consensus to support the proper timing for the postoperative laryngoscopy that is reliable to diagnose RLN palsy. Moreover, it is unknown whether the time interval of postoperative laryngeal inspection plays an important role in the overall rate of RLN injury. We performed a descriptive prospective analysis to assess the impact of different time interval of postoperative VCF assessment on the rate of RLN paralysis diagnosed after thyroidectomy.

\section{Materials and methods}

Starting in 2006, 453 consecutive patients underwent thyroidectomy in a single referral center specialized in endocrine surgery.

Exclusion criteria for this study were preoperative injury to laryngeal nerves and/or intraoperative finding of tumor involvement of RLN.

Main surgical outcome measures were postoperative RLN palsy and follow-up. Patients were followed in collaboration with the Division of Endocrinology and the ENT department of the University of Insubria (Como-Varese), Italy.

Pre- and postoperative follow-up included for all patients FNL to check vocal cord mobility by an independent experienced laryngologist. FNL offers a detailed and wider field of vision to the larynx in a physiological position [9]. The optical illumination and magnification allow the images to be portrayed on a screen for higher definition assessment [9]. The addition of video recording permits repeated evaluations by more than one observer to help interpret subtle findings [9]. Interval comparisons of sequential recordings are useful to evaluate the evolution of the paralysis and the patient's adaptation to it. These assessments are particularly helpful to assess responses to treatment and to direct further intervention. FNL was preferred to videostroboscopy as FNL is at present still the more diffuse available device in all hospitals and with low cost and rapid learning curve when compared with videostroboscopy.

Assessment of time intervals of FNL The postoperative laryngeal examination was outlined according to the following scheme. FNL was performed at 24 to $48 \mathrm{~h}$ before operation (TO), postoperatively, at day 0 in the recovery room (T1) than at second day post-op before patient' hospital discharge (T2) and at +2 weeks (T3) after the surgical procedure. RLN palsy was defined as newly discovered reduction in the movement of the vocal folds after surgery compared with the preoperative status. Frequent laryngoscopy with no documented reason other than determining the complication rate was not supported in this study, i.e., if laryngoscopy was normal at $T 2$ or $T 3$ examination, the patient did not undergo further FNL. For patients with postoperative cord palsy, repeated examinations were performed periodically at $+2(T 4),+6(T 5)$, and +12 months (T6) until full recovery of VCF had been confirmed.

The study and procedure were explained to all patients preoperatively. The surgeon elucidates the patient with vocal cord paralysis, the need for consecutive laryngeal inspection, and the importance of maintaining regular speech therapy for at least 6 months. If 6 months of speech therapy did not result in significant improvement, surgical options were considered $[13,14]$. (It is beyond the scope of this paper to describe these procedures). The protocol and consent were approved by the local institutional review board, and written informed consent was obtained from each patient in advance. Participants were assured anonymity. Included in the study were patients older than 18 years who were scheduled to undergo surgery and were able to provide informed consent. Finally, intraoperative neuromonitoring of laryngeal nerves was used during the study period.

\section{Statistical analysis}

Measurement of the RLN palsy rate was based on the number of nerves at risk (NAR). Data were analyzed for differences in postoperative RLN palsy rate and recovery days.

Unless otherwise stated, all data for continuous variables were expressed as median and range. All patients' data were collected in a prospective manner with a dedicated electronic Microsoft Office Access Data Base (Microsoft Corp, Redmond, WA, USA). Data are collected daily until the day of discharge, then there was a regular contact between the study coordinator and the participating specialist (endocrinologist, laryngologist, etc.) The database is part of our department's quality-improvement program. Primary outcome measure was the morbidity. Statistical analysis was computed with Statistical Package for the Social Sciences (SPSS), release 15.0 for Windows (SPSS Inc, Chicago, IL, USA). The level of significance was set at $P$ less than.05. All efforts were made to avoid sources of bias such as the loss of individuals to follow up during the study. 


\section{Results}

All 453 patients scheduled for thyroidectomy underwent preoperative evaluation of the vocal folds. At preoperative laryngeal examination, we found 11 alterations of the vocal folds movement in 453 patients $(2.4 \%)$. Based on the exclusion criteria, these 11 patients ruled out the analysis.

Complete follow-up was available for $98 \%$ patients (434/442). Four patients were unwilling to accept further laryngeal reexamination, and they were asymptomatic. Moreover, four other individuals were loss during the follow-up study.

Overall, 434 patients appear for postoperative examination of the vocal folds at our university surgical center, providing 825 NAR for examination. Table 1 summarizes the distribution of epidemiological characteristics of patients, thyroid pathology, the proportion of bilateral procedures, mean size of dominant nodules, and mean weight of the thyroid.

The RLN was virtually identified in all cases. No bilateral vocal cord paralysis occurred. Overall permanent RLN palsy occurred in $0.7 \%(N=6)$, while the temporary RLN injury in $6.7 \%(N=56)$. There was no dominance of one side over the other for inferior laryngeal nerve injury $(P>0.05)$.

The rate of RLN morbidity shows a considerable variation due to the different time intervals of FNL. The rate of RLN palsy was $6.4 \%$ at $T 1$ (53 out of 825 NAR), $6.7 \%$ at $T 2$ (56 out of $825 \mathrm{NAR}), 4.8 \%$ at $T 3(N=40), 2.5 \%$ at $T 4(N=21), 0.8 \%$ at $T 5(N=7)$, and $0.7 \%$ at $T 6(N=6)$, according to different time intervals of FNL in the postoperative period. Repeated post-op laryngoscopy examination performed confirmed a dwindling reduction in the incidence of RLN palsy starting at $T 3$ determination. Full recovery of VCF was confirmed after rehabilitation in

Table 1 Preoperative characteristics and operative parameters in 434 patients undergoing thyroidectomy

\begin{tabular}{ll}
\hline & Study group \\
\hline Age (years) & $39.8(19-83)$ \\
Women $(N)$ & 361 \\
Men $(N)$ & 73 \\
Lobectomy & $43(10 \%)$ \\
Total thyroidectomy & $391(90 \%)$ \\
Nerves at risk (NAR) & 825 \\
Preoperative diagnosis & \\
Follicular tumor & $49(11 \%)$ \\
Nodular goiter & $251(58 \%)$ \\
Cancer & $62(14 \%)$ \\
Hyperthyroidism & $72(17 \%)$ \\
\hline
\end{tabular}

Data are numbers or mean with percentages or ranges in parentheses
$49 / 56$ cases at $T 5(87.5 \%)$ and $50 / 56$ at $T 6(89 \%)$. The rate of RLN palsy in $T 2$ was slightly superior than $T 1 . T 1$ and $T 2$ difference did not reach statistical significance, whereas $T 2$ observation was significantly superior to that at $T 3$ in terms of diagnosis of RLN palsy with a noticeably reduction in the rate of nerve palsy at $T 3 \quad(P<0.05)$. Consequently, an inspection of the larynx on the first $48 \mathrm{~h}$ postoperative, before patient' hospital discharge (T2), to identify RLN lesions, is suggested in this analysis. Interestingly, $10.7 \%$ of our patients at $T 2(6 / 56)$ did not see any reason to visit an otolaryngologist because of their normal voice.

\section{Discussion}

This is the first systematic study to evaluate the impact of time interval of the postoperative laryngoscopy for the diagnosis of RLN injury after thyroid surgery. The potential, useful, and proper timing of laryngeal inspection for the diagnosis of RLN palsy is not well documented in literature. This study identifies that the wide variation of rates of RLN palsy are dependent even upon the day of vocal cord assessment in the early postoperative period. The main finding of the present analysis is that FNL at $T 1$ and $T 2$ is nearly similar, with $T 2$ better than $T 1$. Whereas, $T 2$ is significantly superior to that at $T 3$ in terms of diagnosis of RLN palsy with a noticeably reduction in the incidence of nerve palsy at $T 3$. In our experience, the FNL T3 interval evaluation at 2 weeks from operation underestimates RLN injuries and represents incorrect timing for VCF, thus depicting a delay in diagnosis. Consequently, a laryngeal assessment on the first $48 \mathrm{~h}$ postoperative, before patient' hospital discharge, (T2) to identify RLN lesions, is suggested.

The reason of the superior sensitivity of $T 1$ and $T 2$ compared to $T 3$ may be associated with the extent of RLN injury, varying in its severity from neurapraxia to axonotmesis and neurotmesis. In neurapraxia, the structure of the nerve remains intact, but there is a transient interruption in conduction [15]. Neurapraxia is a simple contusion of a nerve with spontaneous return to normal function with rapid recovery over days to months [15]. Axonotmesis is a more significant disruption of the neuronal axon followed by degeneration, but with maintenance of the myelin sheath, and healing takes a prolonged time [15]. Neurotmesis represents a complete division of the nerve and of the encapsulating connective tissue with no spontaneous recovery and requires surgical repair [15]. Studies showed that RLN palsy process subdues from the very first days postoperative [16-19]. Wagner and Seiler, Chiang et al., and Snyder et al. noted that their cases of temporary paralysis resolved in starting early from the third postoperative day [16, 
$18,19]$. Karlan et al. described cases of transient paralysis with recovery ranging from the first week postoperative [17]. In fact, partial injuries (neurapraxia) are much more common than complete transaction (neurotmesis): Snyder et al. and Chiang et al. revealed with the application of intraoperative neuromonitoring that the major cause of RLN injury during thyroid and parathyroid surgery is the traction one which was always a transient injury $[18,19]$. RLN complete transaction was very uncommon in their extensive surgical experience $[18,19]$.

The reason of the slightly superior sensitivity of $T 2$ compared to $T 1$ may be associated to patient's poor compliance, self-adherence, and degree to which he/she correctly follows medical advice during the FNL early after extubation (Tl). Factors that may decrease patient' compliance in $T 1$ include patient feeling ill, limitations of patients activities due to the anesthesia state, acute illness and pain, poor analgesia support, prescription/purpose of FNL not collected/not clear, instructions for FNL not clear, physical difficulty in complying FNL, and unattractive/unpleasant exam.

Authors may raise some concerns on the indication of an FNL in the early postoperative period after thyroidectomy. Other scientist may agree that a postoperative laryngeal inspection is essential in all cases. Most European as well as American thyroid association's guidelines recommend laryngoscopy only for voice dysfunction persisting beyond 2 weeks after thyroidectomy [20,21]. Studies that evaluated the rates of RLN injury after thyroidectomy reported considerable variation in the reported frequency of vocal cord palsy rates due to the different indications to perform laryngoscopy postoperatively (i.e., to all patients or only patients with impaired voice register), moreover, due to different period time (as at extubation, hospital demission, and weeks after surgery) of which we now demonstrated have significant different sensitivities and specificities [6]. All patients need to have pre- and postoperative laryngeal examination if we are to appreciate the true rate at which RLN injury occurs. This study emphasizes the need for postoperative laryngoscopy after thyroidectomy at $T 2$. Without a laryngeal examination, it is impossible to diagnose RLN palsy, and yet the benefits of speech, rehabilitation, steroid, and surgical treatment [13-15, 17]. Certainly, uniform and standard criteria of evaluation of VCF after surgery are needed in order to plan an early and effective treatment to allow comparison of results among different centers and more additional informed postoperative patient discussion.

In this study, permanent RLN palsy occurred in $0.7 \%$ of thyroid surgeries, while the temporary RLN injury is seen in $6.7 \%$. We have noted that approximately $87 \%$ of those nerves that will recover do so within 6 months, $89 \%$ in 12 months. Thus, permanent RLN paralysis may be diagnosed only on the basis of serial laryngeal examinations between 6 and 12 months after thyroid surgery. Therefore, we suggest that a minimum follow-up period of 12 months is needed to assess voice function after thyroidectomy if dysphonia is detected. This is in concordance with other studies where the most commonly adopted and recommended period of follow-up for RLN palsy after thyroid surgery was 1 year [13-15, 17, 22].

\section{Conclusion}

In conclusion, we report different interval time evaluation criteria of vocal cord motility post-thyroid surgery with great and significant variability of results. The rate of RLN palsy is influenced by the "timing" of the postoperative laryngoscopy. The proper timing of laryngeal inspection for the diagnosis of RLN palsy is at second day post-op.

Competing interest The authors declare that they have no competing interest.

Authors' contributions GD, PC, and RD have made substantial contributions to acquisition of data; FR and LB contributed to study conception and design; GD contributed to analysis and interpretation of data; $\mathrm{LB}$ and RD contributed to drafting of manuscript; and RD contributed to critical revision and supervision.

\section{References}

1. Brok HA, Copper MP, Stroeve RJ, Ongerboer de Visser BW, Venker-van Haagen AJ, Schouwenburg PF (1999) Evidence for recurrent laryngeal nerve contribution in motor innervation of the human cricopharyngeal muscle. Laryngoscope 109(5):705-708

2. Lahey FH, Hoover WB (1938) Injuries to the recurrent laryngeal nerve in thyroid operations: their management and avoidance. Ann Surg 108(4):545-562

3. Hermann M, Alk G, Roka R, Glaser K, Freissmuth M (2002) Laryngeal recurrent nerve injury in surgery for benign thyroid diseases: effect of nerve dissection and impact of individual surgeon in more than 27,000 nerves at risk. Ann Surg 235(2):261268

4. Thomusch O, Machens A, Sekulla C (2000) Multivariate analysis of risk factors for postoperative complications in benign goiter surgery: prospective multicenter study in Germany. World J Surg 24(11):1335-1341

5. Barczyński M, Konturek A, Cichoń S (2009) Randomized clinical trial of visualization versus neuromonitoring of recurrent laryngeal nerves during thyroidectomy. Br J Surg 96(3):240-246

6. Bergenfelz A, Jansson S, Kristoffersson A, Mårtensson H, Reihnér E, Wallin G, Lausen I (2008) Complications to thyroid surgery: results as reported in a database from a multicenter audit comprising 3,660 patients. Langenbeck's Arch Surg 393(5):667-673

7. Randolph GW (ed) (2003) Surgical anatomy of the recurrent laryngeal nerve. In: Surgery of the thyroid and parathyroid glands. Elsevier Science, Philadelphia 
8. Randolph GW, Kamani D (2006) The importance of preoperative laryngoscopy in patients undergoing thyroidectomy: voice, vocal cord function, and the preoperative detection of invasive thyroid malignancy. Surgery 139(3):357-362

9. Echternach M, Maurer CA, Mencke T, Schilling M, Verse T, Richter B (2009) Laryngeal complications after thyroidectomy: is it always the surgeon? Arch Surg 144(2):149-153

10. Jeannon JP, Orabi AA, Bruch GA, Abdalsalam HA, Simo R (2009) Diagnosis of recurrent laryngeal nerve palsy after thyroidectomy: a systematic review. Int J Clin Pract 63(4):624629

11. Farrag TY, Samlan RA, Lin FR, Tufano RP (2006) The utility of evaluating true vocal fold motion before thyroid surgery. Laryngoscope 116(2):235-238

12. Schlosser K, Zeuner M, Wagner M, Slater EP, Domínguez Fernández E, Rothmund M, Maschuw K (2007) Laryngoscopy in thyroid surgery: essential standard or unnecessary routine? Surgery 142(6):858-864

13. Miller S (2004) Voice therapy for vocal fold paralysis. Otolaryngol Clin North Am 37(1):105-119

14. Laccourreye O, Papon JF, Kania R, Crevier-Buchman L, Brasnu D, Hans S (2003) Intracordal injection of autologous fat in patients with unilateral laryngeal nerve paralysis: long-term results from the patient's perspective. Laryngoscope 113(3):541-545

15. Seddon H (1965) Nerve injuries. Med Bull (Ann Arbor) 31:4-10
16. Wagner HE, Seiler C (1994) Recurrent laryngeal nerve palsy after thyroid gland surgery. Br J Surg 81(2):226-228

17. Karlan MS, Catz B, Dunkelman D, Uyeda RY, Gleischman S (1984) A safe technique for thyroidectomy with complete nerve dissection and parathyroid preservation. Head Neck Surg 6 (6):1014-1019

18. Chiang FY, Lu IC, Kuo WR (2008) The mechanism of recurrent laryngeal nerve injury during thyroid surgery-the application of intraoperative neuromonitoring. Surgery 143(6):743-749

19. Snyder SK, Lairmore TC, Hendricks JC, Roberts JW (2008) Elucidating mechanisms of recurrent laryngeal nerve injury during thyroidectomy and parathyroidectomy. J Am Coll Surg 206 (1):123-130

20. Watkinson JC, British Thyroid Association (2004) The British Thyroid Association guidelines for the management of thyroid cancer in adults. Nucl Med Commun 25(9):897-900

21. Cooper DS, Doherty GM, Haugen BR, Kloos RT, Lee SL, Mandel SJ, Mazzaferri EL, McIver B, Sherman SI, Tuttle RM, American Thyroid Association Guidelines Taskforce (2006) Management guidelines for patients with thyroid nodules and differentiated thyroid cancer. Thyroid 16(2):109-142

22. Kelchner LN, Stemple JC, Gerdeman E, Le Borgne W, Adam S (1999) Etiology, pathophysiology, treatment choices, and voice results for unilateral adductor vocal fold paralysis: a 3-year retrospective. J Voice 13(4):592-601 